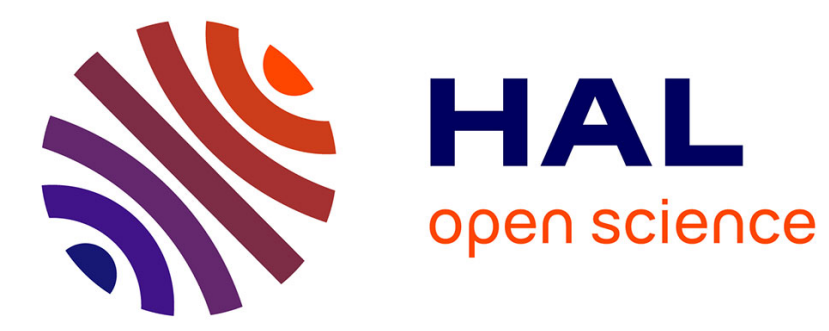

\title{
Aimants supraconducteurs pour la physique corpusculaire
}

H. Desportes

\section{To cite this version:}

H. Desportes. Aimants supraconducteurs pour la physique corpusculaire. Revue de Physique Appliquée, 1969, 4 (2), pp.233-234. 10.1051/rphysap:0196900402023300 . jpa-00243236

\section{HAL Id: jpa-00243236 https://hal.science/jpa-00243236}

Submitted on 1 Jan 1969

HAL is a multi-disciplinary open access archive for the deposit and dissemination of scientific research documents, whether they are published or not. The documents may come from teaching and research institutions in France or abroad, or from public or private research centers.
L'archive ouverte pluridisciplinaire HAL, est destinée au dépôt et à la diffusion de documents scientifiques de niveau recherche, publiés ou non, émanant des établissements d'enseignement et de recherche français ou étrangers, des laboratoires publics ou privés. 


\title{
AIMANTS SUPRACONDUGTEURS POUR LA PHYSIQUE GORPUSGULAIRE
}

\author{
H. DESPORTES, \\ D.Ph.P.E., G.E.N., Saclay.
}

\begin{abstract}
Résumé. - L'état actuel des techniques d'aimants supraconducteurs est brièvement indiqué, ainsi que quelques applications réalisées ou envisagées en physique corpusculaire. L'exemple de l'aimant "Héra " pour cible polarisée est mentionné.
\end{abstract}

\begin{abstract}
The present status of superconducting magnets is briefly reviewed, in view of existing or possible applications in high energy physics. An example is given in the polarized target "Hera" magnet.
\end{abstract}

Le champ d'application le plus étendu des aimants supraconducteurs est à l'heure actuelle la physique des particules, domaine qui fait un usage extensif des champs magnétiques et dont l'équipement expérimental le plus spectaculaire est celui des grandes chambres à bulles. Les techniques d'aimants supraconducteurs ont atteint à présent un stade de développement suffisamment sûr pour que leur emploi puisse être étendu à une gamme importante d'applications. A titre d'exemple, indiquons que l'on sait produire couramment des champs magnétiques de 100 à $150 \mathrm{kG}$ dans de faibles volumes (jusqu'à $20 \mathrm{~cm}$ ), des champs de 50 à $80 \mathrm{kG}$ dans des volumes de l'ordre du mètre, et que l'on peut envisager des aimants de plusieurs mètres avec des champs de 20 à $30 \mathrm{kG}$. Ces performances peuvent être obtenues dans des conditions de fonctionnement tout à fait opérationnelles, c'est-à-dire avec des caractéristiques de stabilité, de reproductibilité, de mise en charge et de sécurité très satisfaisantes.

Pour une description détaillée des problèmes et des solutions techniques, on peut se reporter aux références [1].

Les réalisations concrètes en physique des particules ont porté en premier lieu sur des applications où les techniques conventionnelles atteignaient leurs limites techniques. G'est le cas des très grandes chambres à bulles dans lesquelles les volumes de champ à créer sont considérables et nécessitent des puissances électriques extrêmement élevées. Il existe trois projets de grandes chambres à hydrogène à aimants supraconducteurs en cours de réalisation : la chambre de $7 \mathrm{ft}$ de Brookhaven, dont les bobines ont 2,4 $\mathrm{m}$ de diamètre utile et doivent produire $30 \mathrm{kG}$, soit $40 \mathrm{MJ}$ d'énergie magnétique; la chambre de $12 \mathrm{ft}$ d'Argonne, dont les bobines atteignent $4,8 \mathrm{~m}$ de diamètre avec un champ de $20 \mathrm{kG}$, et $80 \mathrm{MJ}$ d'énergie magnétique; enfin la chambre européenne de $3,7 \mathrm{~m}$, dont les bobines auront $4,7 \mathrm{~m}$ de diamètre avec un champ de $35 \mathrm{kG}$ et $750 \mathrm{MJ}$ d'énergie magnétique.
Une autre application qui a suscité très vite un intérêt vis-à-vis des supraconducteurs est celle des aimants de cibles polarisées. Ces aimants doivent remplir des conditions très rigoureuses : champ magnétique de 18,25 ou $50 \mathrm{kG}$ suivant le type de cible; uniformité du champ à $10^{-4}$ près sur la cible; grande accessibilité à la cible pour le passage des faisceaux, en particulier dans la direction du champ magnétique. Un aimant de ce type ( $f g .1$ ) a été réalisé au D.Ph.P.E. à Saclay et se trouve en exploitation depuis fin 1967 dans l'expérience « Héra » auprès du PS au C.E.R.N. Les caractéristiques de cet aimant ont été décrites

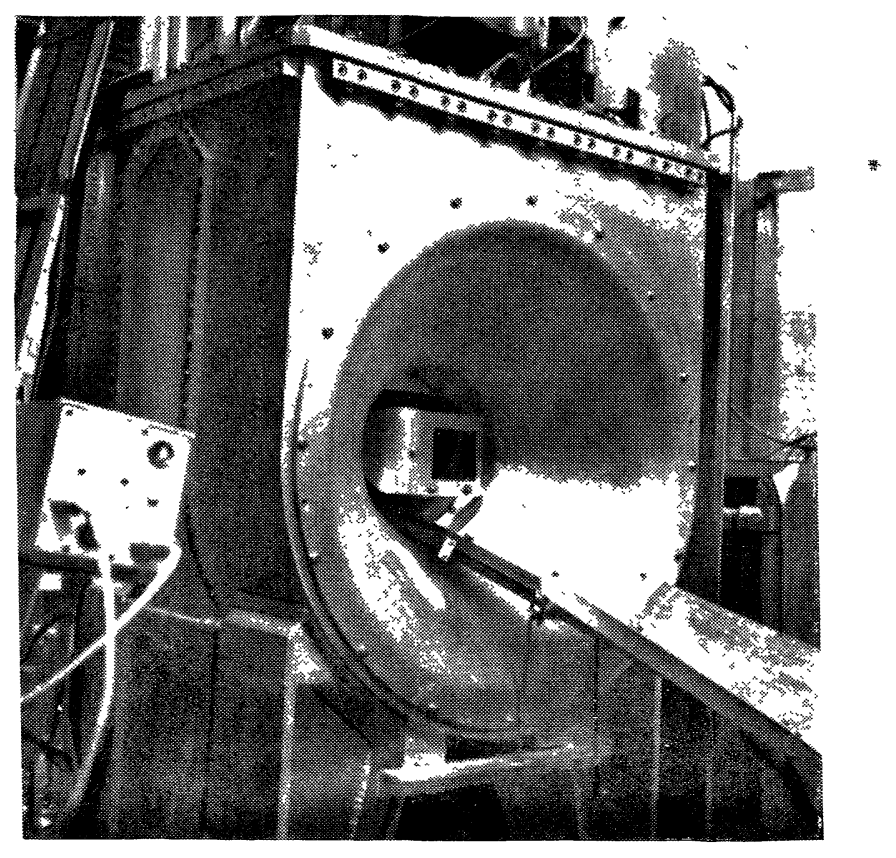

FIG. 1.

Aimant "Héra " en place dans l'expérience. 
par ailleurs [2]. Indiquons seulement qu'il est constitué de deux bobines sans fer en position de Helmholtz, de $40 \mathrm{~cm}$ de diamètre intérieur, produisant un champ maximal de $26 \mathrm{kG}$, correspondant à une énergie magnétique de $400 \mathrm{~kJ}$. Pour son fonctionnement, l'aimant est associé à un réfrigérateur à hélium liquide et est relié électriquement en permanence à une alimentation stabilisée; le temps d'établissement du champ est de l'ordre de quinze minutes, la coupure normale se fait en trois minutes et les dispositifs de protection permettent une décharge rapide en vingt secondes.

Bien que les exemples précédents représentent les seules applications effectivement mises en œuvre jusqu'à présent dans des équipements expérimentaux de physique corpusculaire, de nombreuses autres applications sont envisagées : aimants de chambre à étincelles, spectromètres et surtout aimants de faisceaux, déviateurs et quadrupôles. Ce dernier type d'appareil fait l'objet d'études poussées dans de nombreux laboratoires et, dans un délai d'une ou deux années, il est probable que plusieurs dispositifs seront mis en place dans des faisceaux. On peut cependant remarquer que l'utilisation de tels aimants, à part quelques cas très particuliers, ne répond pas à un besoin impératif auprès des accélérateurs actuels et que les études en cours sont sans aucun doute stimulées par la perspective des futurs accélérateurs à très haute énergie. Aux ÉtatsUnis, l'accélérateur national de 200-400 GeV entre actuellement dans sa phase de réalisation et les études d'aimants supraconducteurs occupent une place privilégiée dans la préparation des équipements expérimentaux. Il n'est pas exclu que ces nouvelles techniques ne soient généralisées à l'ensemble des quelque 300 aimants prévus auprès d'une machine de cette taille. Ge qu'il est important de constater, c'est qu'un tel choix ne repose plus seulement sur des possibilités techniques, mais principalement sur un bilan économique.

\section{BIBLIOGRAPHIE}

[1] LAVERICK (Ch.), Superconducting Magnet Technology, Part I : Advances in Electronics and Electron Physics, 1963, vol. 23 (Academic Press, Inc., New York). Part II : Report ANL/HEP 6810.

[2] Desportes (H.), Proc. of the 2nd Intern. Conf. on Magnet Technology, Oxford, 1967, p. 603. 